\title{
Crosstalk between androgen and Wnt/ $\beta$-catenin leads to changes of wool density in FGF5-knockout sheep
}

\author{
Rui Zhang $\mathbb{1}^{1}$, Yan $\mathrm{Li}^{1}$, Kun Jia ${ }^{1}$, Xueling Xu', Yao Li', Yue Zhao ${ }^{1}$, Xiaosheng Zhang ${ }^{2}$, Jinlong Zhang ${ }^{2}$, Guoshi Liu', \\ Shoulong Deng ${ }^{3}$ and Zhengxing Lian ${ }^{1}$
}

\begin{abstract}
Fibroblast growth factor 5 (FGF5) is a famous dominant inhibitor of anagen phase of hair cycle. Mutations of FGF5 gene result in a longer wool in mice, donkeys, dogs, cats, and even in human eyelashes. Sheep is an important source of wool production. How to improve the production of wool quickly and effectively is an urgent problem to be solved. In this study, we generated five FGF5-knockout Dorper sheep by the CRISPR/Cas9 system. The expression level of FGF5 mRNA in knockout (KO) sheep decreased significantly, and all FGF5 proteins were dysfunctional. The KO sheep displayed a significant increase in fine-wool and active hair-follicle density. The crosstalk between androgen and Wnt/ $\beta$-catenin signaling downstream of FGF5 gene plays a key role. We established downstream signaling cascades for the first time, including FGF5, FGFRI, androgen, AR, Wnt/ $\beta$-catenin, Shh/Gli2, c-MYC, and KRTs. These findings further improved the function of FGF5 gene, and provided therapeutic ideas for androgen alopecia.
\end{abstract}

\section{Introduction}

Hair is a primary characteristic of mammals. Hair-follicle development takes place during fetal skin development, and relies on tightly regulated ectodermal-mesodermal interactions. After birth, mature and actively growing hair follicles eventually become anchored in the subcutis, and periodically regenerate by spontaneously undergoing repetitive cycles. The hair-growth cycle in mammals is composed of three phases: anagen, catagen, and telogen ${ }^{1-4}$. Many of the signaling cascades involved in embryonic hair development are redeployed during adult hair development, and hyperactivation or inappropriate maintenance of these signaling pathways can result in skin disorders and cancers.

\footnotetext{
Correspondence: Shoulong Deng (popo84350746@163.com) or

Zhengxing Lian (lianzhx@cau.edu.cn)

'Beijing Key Laboratory of Animal Genetic Improvement, National Engineering Laboratory for Animal Breeding, Key Laboratory of Animal Genetics and Breeding of the Ministry of Agriculture, College of Animal Science and Technology, China Agricultural University, Beijing 100193, China

${ }^{2}$ Tianjin Institute of Animal Sciences, Tianjin 300112, China

Full list of author information is available at the end of the article

Edited by E. Cand
}

The FGF5 gene is a member of the FGF family, which exhibits a diverse array of biological activities ${ }^{5}$. Hebert et al. ${ }^{6}$ reported that deletion of the FGF5 gene abnormally prolongs anagen in mice, suggesting that its expression leads to termination of anagen and induction of catagen. Subsequent to identification of FGF5 as causative for the angora mouse phenotype, genetic variants in FGF5 have been shown to underlie hair-length regulation in several other species, including cats (Felis catus) ${ }^{7,8}$, dogs (Canis lupus familiaris $^{9,10}$, donkeys (Equus africanus asinus) $)^{11}$, Syrian hamster

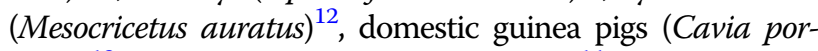
cellus) ${ }^{13}$, and even human (Homo sapiens) ${ }^{14}$.

Sheep are one of the most important wool animals. However, traditional breeding limits the genetic improvement of sheep breeding in the short term. Current results indicate that the CRISPR-Cas9 system can be successfully employed in a broad range of organisms ${ }^{15-21}$. In the previous study, we had successfully generated FGF5-knockout heterozygous sheep using the CRISPR/ Cas9 system by direct one-step cytoplasmic injection of Cas9 mRNA and sgRNA into zygotes, and the relationship

\section{(c) The Author(s) 2020}

(c) (i) Open Access This article is licensed under a Creative Commons Attribution 4.0 International License, which permits use, sharing, adaptation, distribution and reproduction c. in any medium or format, as long as you give appropriate credit to the original author(s) and the source, provide a link to the Creative Commons license, and indicate if changes were made. The images or other third party material in this article are included in the article's Creative Commons license, unless indicated otherwise in a credit line to the material. If material is not included in the article's Creative Commons license and your intended use is not permitted by statutory regulation or exceeds the permitted use, you will need to obtain permission directly from the copyright holder. To view a copy of this license, visit http://creativecommons.org/licenses/by/4.0/. 
between FGF5 gene and wool length in sheep was proved $^{22}$. In this study, we continue the previous approach, and found that FGF5 is also related to the wool and active hair-follicle density in Dorper sheep. However, the specific mechanism of the FGF5 gene in the development of hair follicles is unclear, and whether it has other effects besides promoting changes in the follicular cycle is currently elusive.

Androgenetic alopecia (AGA) is the most common form of hair loss in humans, which is mediated mainly by androgens. Androgens regulate hair growth, sebum production, and secretion, among other physiological effects in the $\operatorname{skin}^{23,24}$. Androgen levels are under the control of enzymes. Testosterone, as one of the androgens, can be reduced to dihydrotestosterone (DHT) by $5 \alpha$-reductase (SRD5A) enzyme, which has three isotypes. SRD5A1 is predominantly expressed in skin and annexes ${ }^{23,25,26}$. Hydroxysteroid 17-beta-dehydrogenase 2 (HSD17 $\beta 2$ ) is also a key player in the inactivation of testosterone ${ }^{27}$. The importance of the Wnt/B-catenin pathway in AGA is emphasized by the demonstration of molecular crosstalk between androgens and $\mathrm{Wnt}$ signaling in dermal papilla cell $(\mathrm{DPC})^{28}$. Androgen/AR complex binding to antioxidative response elements (AREs) containing promoters of target genes disrupts Wnt agonist/antagonist balance involved in DPC-inductive ability, such as dickkopf-1 (DKK1), which is a specific inhibitor of Wnt coreceptors of the LRP family ${ }^{29}$.

Sonic hedgehog (Shh) also plays an important role in both embryonic and adult hair development. Lim et al. ${ }^{30}$ showed that hair-follicle activation in Wnt-active dermal cells promotes their fate conversion into dermal papilla (DP), the regenerative dermal niche for hair-follicle formation. Also, Veltri et al. ${ }^{31}$ believed that Shh signaling serves as a downstream pathway of Wnt/ $\beta$-catenin signaling to regulate hair-follicle (HF) induction.

Is there an association between the increase of wool and active hair-follicle density in FGF5 KO sheep and AGA? If so, does crosstalk between AR and Wnt/ $\beta$-catenin also participate in the process of wool and active hair-follicle density in FGF5 KO sheep? Are there any other signaling pathways or other factors that also play a role? In this study, we revealed that the crosstalk between androgen and Wnt/B-catenin signaling plays a major role in the increase in wool and active hair-follicle density due to the activation of $c-M Y C$ and $K R T S$ associated with inner root sheath (IRS) in FGF5 KO sheep, and the Shh pathway is also involved in this process as a downstream pathway of $W n t / \beta$-catenin signaling.

\section{Results}

\section{Generation and screening of FGF5 KO sheep}

Since we have investigated the efficiency of detecting mutations by PCR sequencing (Supplementary Table S1), the blood DNA template was screened for positive individuals using PCR and DNA sequencing (Supplementary
Fig. S1). As expected, there were a total of eight mutants in five founders (including three females and two males) (Fig. 1c), and one mutation appeared in three of the founders.

Cas9 may induce a troublesome off-target effect ${ }^{32}$. A total of 12 most potential off-target sites were predicted across the sheep genome. The sequencing results indicated that there was no detectable indel at on-target sites (Supplementary Fig. S2).

\section{Structure of FGF5 protein and expression level have changed resulting from gene editing}

Sequencing results demonstrated that mosaicism existed in four founders. The indels result in a FGF5 protein frameshift or deletion of AA (Fig. 1d), and the $\beta$ 12 strands were destroyed or changed. The 3D models also predicted that the mutated FGF5 lacked the $\beta$ 12 strands that define the canonical trefoil of the FGF family (Fig. 1e).

The results of the transcription and the protein level of FGF5 expression demonstrated that intact FGF5 mRNA was significantly downregulated $(P<0.01)$ in the $\mathrm{KO}$ group compared with the control group (Figs. 1f and $3 \mathrm{~b}$ ); the intact FGF5 protein was significantly downregulated $(P<0.05)$ as well (Fig. 1g).

\section{Wool and active follicle density have increased due to gene editing}

To investigate the changes in wool phenotype caused by the loss-of-function mutation in FGF5, the density and fineness of the wool were measured from three parts of both the $\mathrm{KO}$ and control group (Fig. 2a). Both the $\mathrm{KO}$ and the control group have three types of wool hair, including medullated wool, heterotypical wool, and nonmedullated wool, and the fineness of three types of wool hair has no significant change, except for the medullated wool in the hindquarter $(P<0.01$, Fig. $2 \mathrm{~b}, \mathrm{c})$. The coarse-wool densities of the $\mathrm{KO}$ group were significantly lower than the control group in the anterior shoulder and body side, while the fine-wool densities of the $\mathrm{KO}$ group were significantly higher than the control group in all three parts $(P<0.001$, Fig. 2 d, e). Sacpic staining showed that the proportions of total, primary, and secondary active hair follicles in the $\mathrm{KO}$ group, were significantly higher than that in the control group. (Fig. 2f, g). Changes in sheep wool and active hair-follicle density after FGF5 gene editing in catagen and telogen were also investigated (Supplementary Fig. S3). In catagen, the coarse-wool densities have no significant change, while the fine-wool densities of the $\mathrm{KO}$ group were significantly higher than the control group in all three parts $(P<0.05$, Fig. S3a-c). Sacpic staining results also showed that the proportion of primary active hair follicles has no significant change, while the proportion of secondary active hair follicles in 


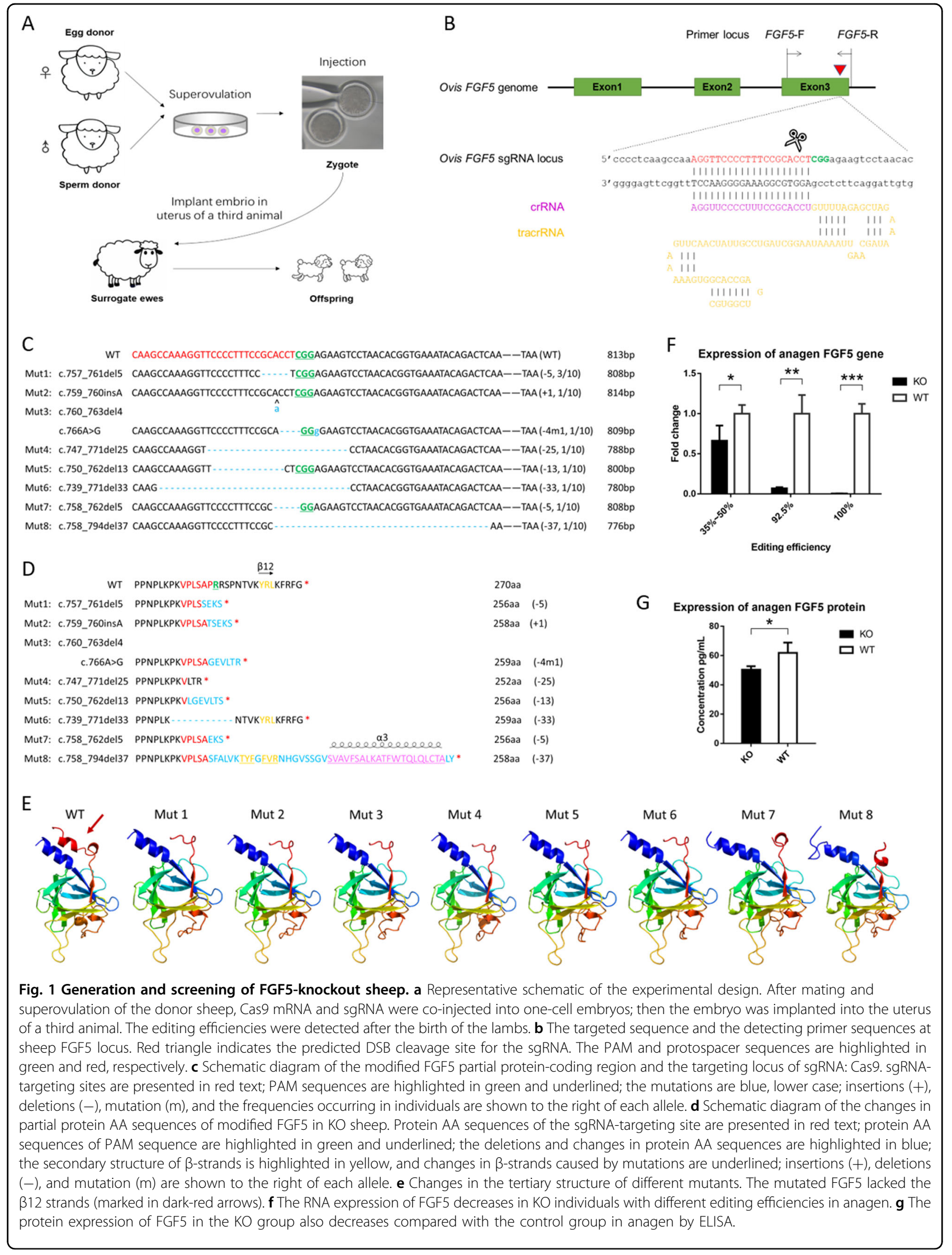




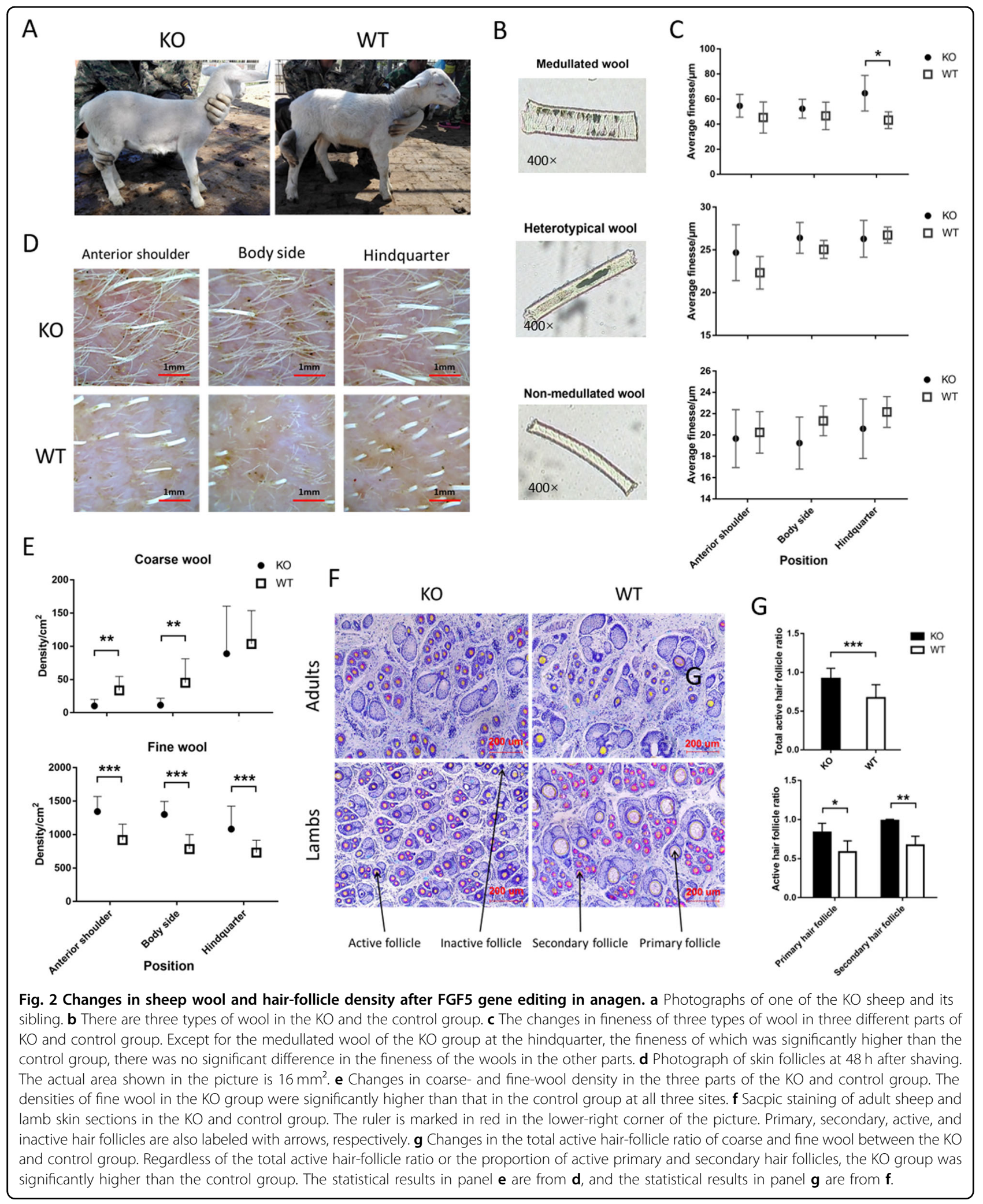

the $\mathrm{KO}$ group was significantly higher than that in the control group. In telogen, the densities of coarse and fine wool have no significant change, except that the fine-wool density in the $\mathrm{KO}$ group was significantly higher in the anterior shoulder than that in the control group $(P<0.01)$. Sacpic staining results also showed that the proportion of 
primary and secondary active hair follicles has no significant change.

\section{Androgen receptor and Wnt signaling operate as key stimulators of HF development in FGF5 KO sheep}

To explore the causes of increased wool and active hair-follicle density in FGF5 KO sheep, we tested the expression levels of testosterone and DHT that cause androgenetic alopecia in the skin tissue of the $\mathrm{KO}$ and control groups by ELISA. As a result, the levels of testosterone in the $\mathrm{KO}$ and control group were comparable, while the level of DHT was significantly reduced in the KO group (Fig. 3a). The mRNA and the protein levels of SRD5A1, AR, and DKK1 were significantly lower in the $\mathrm{KO}$ than in the control group $(P<0.05)$, while $17 \beta-H S D 2$ mRNA level was significantly higher in the $\mathrm{KO}$ group (Fig. 3c). Besides, the protein level of $\beta$-catenin was indeed significantly higher in the $\mathrm{KO}$ group (Fig. 3f, g). At this point, we were sure that the crosstalk between androgen and $W n t / \beta$-catenin signaling was involved in this process.

Based on these results, we have also explored the pathways downstream of $\mathrm{Wnt} / \beta$-catenin signaling. As a result, the expression levels of KRT27 and KRT28, which are essential for the proper assembly of type I and II keratin protein complexes and formation of keratin intermediate filaments in the IRS, were significantly higher in the KO group (Fig. 3d). The expression level of c-MYC was significantly higher in the KO group (Fig. 3f, g). The expression levels of other related genes are shown in Supplementary Fig. S4. In the Wnt signaling pathway, expression levels of Wnt-10a, Wnt-10b, LRP6, Lef1, and $\beta$-catenin were comparable in the $\mathrm{KO}$ and control group. The same as related factors in the Wnt signaling pathway, expression levels of related factors in the BMP signaling pathway, such as $B M P 2, B M P 4, B M P 7$, and Samd2, and expression levels of other related factors, such as $F G F 7$, CYP17, CXCL13, EDAR, TGF 2 2, PI3KB, and Akt, were also comparable.

\section{Shh signaling participates in the process of wool and active hair-follicle density increase through GLI2}

Sonic hedgehog (Shh) signaling plays a critical role in hair-follicle development and skin cancer. Since we have previously confirmed the involvement of $W n t / \beta$-catenin signaling, as a factor downstream of the $W n t / \beta$-catenin signaling pathway, we are also curious about the role of the Shh pathway in the increase of wool and active hairfollicle density. The same method as before, we examined the transcription of Shh, Gli1, Gli2, and Gli3. As a result, Shh mRNA level was significantly lower in the KO than in the control group $(P<0.05)$, Gli2 mRNA level was significantly higher in the $\mathrm{KO}$ group, and the expression levels of Gli1 and Gli3 had no significant change (Fig. 3e).
As Mill et al. ${ }^{33}$ demonstrated that Gli2 is the key mediator of Shh responses in the skin, and Gli2 controls epithelial cell proliferation in embryonic skin through transcriptional regulation of cyclin D1 and D2, we believed that Shh signaling participated in the process of active hairfollicle density increase through Gli2. c-MYC, whose expression level was significantly higher in the $\mathrm{KO}$ group, could also be activated by Shh.

\section{Addition of DHT and finasteride to DPCs demonstrates the interaction between androgen and Wnt/ $\beta$-catenin}

Since the crosstalk between androgen and Wnt/ $\beta$-catenin pathway plays a pivotal role in the regulation of hair growth, the effects of DHT and finasteride on the levels of $A R$ and $\beta$-catenin were examined in the DPCs. DPCs were obtained from sheep skin tissue by isolation and purification (Fig. 4a, b), and identified by immunofluorescence staining (Fig. 4c). The positive $\alpha$-SMA proved to be DPCs, and CD133-positive proved that the DPCs were in the growth phase. When the DPCs were treated with $10^{-7} \mathrm{M}$ DHT for $48 \mathrm{~h}$, the level of $A R$ in the DPCs of the KO group increased significantly, and the level of DKK1 in the DPCs of the KO and control groups all increased significantly. When the DPCs were treated with $10^{-5} \mathrm{M}$ finasteride for $48 \mathrm{~h}$, the levels of $A R$ and $D K K 1$ in the DPCs of the control group all decreased significantly (Fig. 4d). The concentrations of DHT and finasteride used were selected based on the results of previous experiments, as shown in Supplementary Fig. S5. Furthermore, regardless of the treatment, the expression levels of the $A R$ and $D K K 1$ gene in the KO group were always significantly lower than those in the control group. Similar results were obtained by western blot analysis of AR, DKK1, and $\beta$-catenin (Fig. 4e).

\section{Discussion}

CRISPR-Cas9 is an RNA-guided gene-editing tool that offers several advantageous characteristics in comparison with the conventional methods (e.g., zinc finger nucleases and transcription activator-like effector nucleases). Despite some limitations, such as efficient delivery and safety, CRISPR-Cas9 is still the most convenient tool for gene-editing purposes ${ }^{34,35}$. There were reports on the lengthening of the wool after the knockdown of the FGF5 gene in sheep and goats ${ }^{22,36,37}$. However, there are no reports on the effects of FGF5 gene on wool and hairfollicle density, except Wang et al. ${ }^{37}$. Here, we confirmed in Dorper sheep that the FGF5 gene affects wool and active hair-follicle density, and that FGF5 KO sheep have more fine wool and active secondary hair follicles.

Activation of the $W n t / \beta$-catenin signaling pathway is important for the initiation and maintenance of hair morphogenesis ${ }^{29,38}$, and is critical for the maintenance of DPC-inductive properties required for hair-follicle 

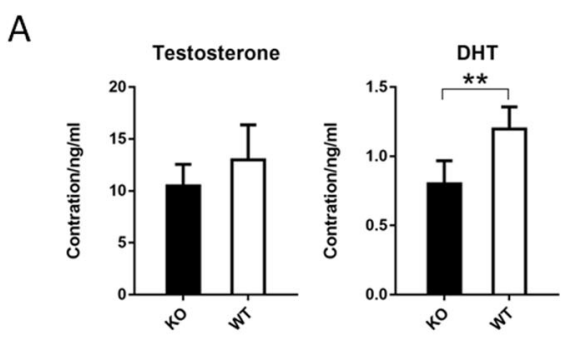

B
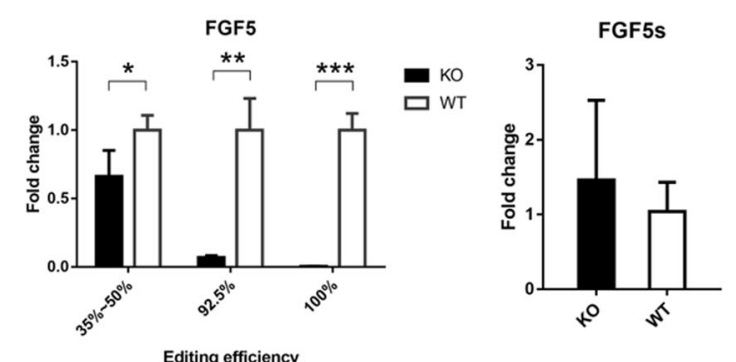

C
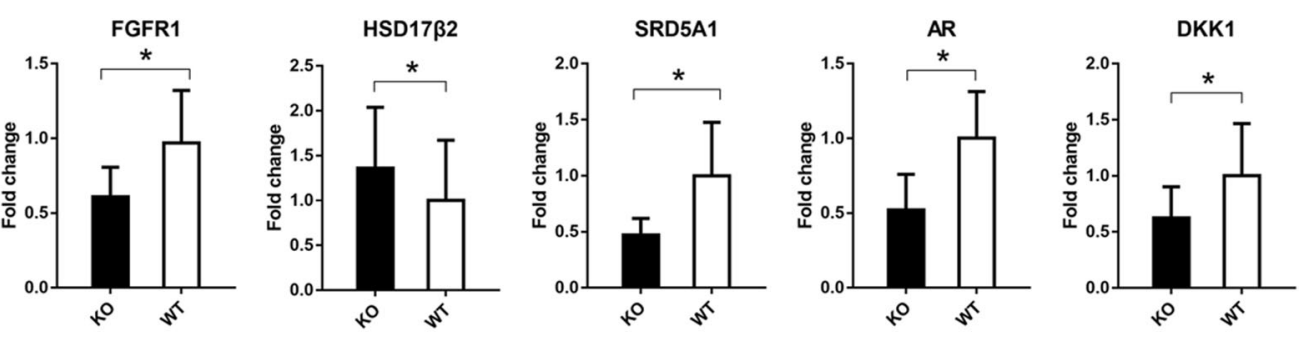

D
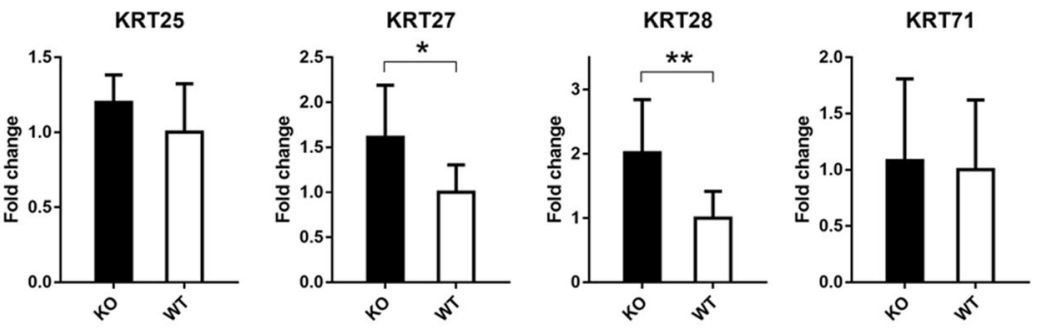

E
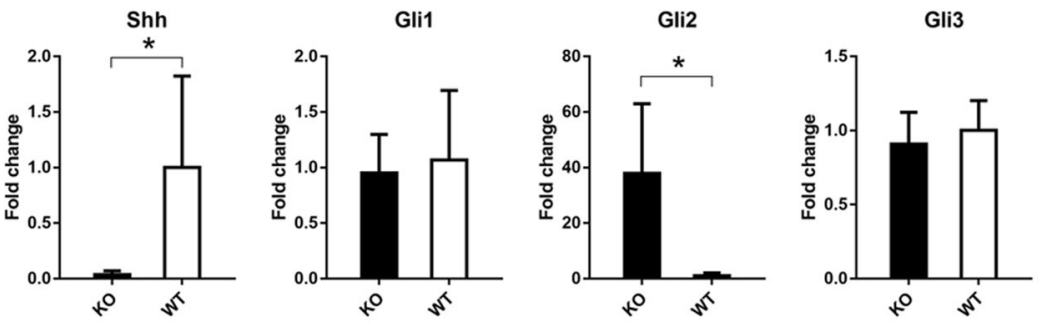

G
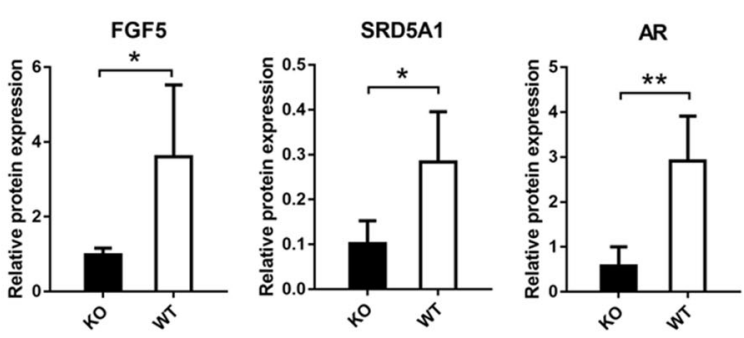

F
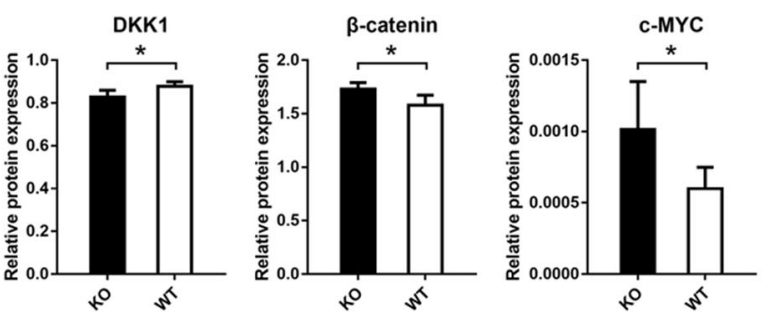

Fig. 3 (See legend on next page.) 
(see figure on previous page)

Fig. 3 FGF5-mediated multiple signaling promotes the increase in wool density and the active hair-follicle density in anagen. a Changes in testosterone and DHT in the skin tissue of KO and control groups. The expression level of DHT in the skin tissue of the KO group was significantly lower than that of the control group. $\mathbf{b}$ Changes in FGF5 in the skin tissue of the KO and control group. The expression levels of FGF5 gene in different editing efficiency individuals were different, but they were significantly lower than the control group. However, the expression level of the FGF5s gene did not change significantly. c Expression changes of the related genes in AR and Wnt signaling in the skin tissue of the KO and control group. $\mathbf{d}$ Expression changes of IRS-related KRTs in the skin tissue of the KO and control group. e Expression changes of Shh signaling in the skin tissue of the KO and control group. f The expression levels of FGF5, SRD5A1, AR, DKK1, $\beta$-catenin, and c-MYC proteins in the skin tissue were detected by western blotting. The amount of $\beta$-tubulin was used as a control. $\mathbf{g}$ Compared with the control group, the protein expression levels of FGF5, SRD5A1, AR, DKK1, $\beta$-catenin, and C-MYC in the KO group were significantly changed.

regeneration and growth of the hair shaft ${ }^{39,40}$. Previous research had shown that in DP cells from AGA patients, the $W n t / \beta$-catenin signaling pathway is negatively influenced by ligand-activated AR, inhibiting HFSC differentiation $^{28}$. DHT-inducible DKK1 produced by balding DP cells promotes apoptosis of neighboring follicular keratinocytes in vitro, together with the higher expression of DKK1 in balding compared with the haired scalp, suggesting that $D K K 1$ may be one of the key factors involved in the pathogenesis of $\mathrm{AGA}^{41}$. In the present study, we associate the phenotype of increased wool density with the phenotype of AGA, and tests of testosterone and DHT speculated that androgen-induced signaling pathways were highly likely to be involved. Thus, we focused on whether the Wnt/ $\beta$-catenin signaling pathway was involved. The expression of $\beta$-catenin protein in the skin tissues of the $\mathrm{KO}$ group was significantly higher than in the control group. $D K K 1$ were significantly lower in the $\mathrm{KO}$ group in both the mRNA and protein levels. For further verification of the crosstalk between androgen and $W n t / \beta$-catenin pathway, we added DHT, finasteride, and DMSO in the culture medium of DPCs to detect the expression of related genes. We explored the optimal concentration of DHT and finasteride in DPCs based on relevant reports $^{42}$ (Supplementary Fig. S5). The results showed that when the DPCs were treated with $10^{-7} \mathrm{M}$ DHT for $48 \mathrm{~h}$, the level of $A R$ in the DPCs of the KO group increased significantly, while the level of $A R$ in the DPCs of the control group decreased significantly. We still have not got a reasonable explanation for the decrease of $A R$ in the DPCs of the control group; however, it seems to be related to the amount of DHT contained in the sample itself. Since the control group itself contains more DHT, it requires more exogenous DHT to function. When the DPCs were treated with $10^{-5} \mathrm{M}$ finasteride for $48 \mathrm{~h}$, the levels of $A R$ and $D K K 1$ in the DPCs of the control group all decreased significantly, while the levels of $A R$ and $D K K 1$ in the DPCs of the $\mathrm{KO}$ group decreased without significance. This may be due to the fact that the expression level of SRD5A1 in the $\mathrm{KO}$ group is already very low, and finasteride has a limited inhibitory effect compared with the control group.

Besides, the relationship between FGFR1 and androgen has been reported in studies of the transition of hormonenaive to castrate-resistant prostate cancer ${ }^{43}$. However, the relationship between FGFR1 and androgen in hair follicles needs further exploration and improvement.

Finally, based on the increase in wool and active hairfollicle density in FGF5 KO sheep, we first mapped out the main mechanism of FGF5 function in anagen, including FGF5, FGFR1, androgen, AR, Wnt/ $\beta$-catenin, c-MYC and KRTs, and Shh/GLI2 (Fig. 5), which helps to further understand the role of FGF5 gene, and provide a treatment for androgen alopecia. Of course, we also tested the genes of other related signaling pathways (Supplementary Fig. S6). However, there are still more details that require further research and enrichment, such as the interaction of Wnt-BMP, Noggin-Shh, WntNotch, and so on.

\section{Materials and methods \\ Ethics statement}

All animal experimental procedures in this study were approved and carried out in strict accordance with guidelines for the care and use of animals of the Animal Care and Use Committee at China Agricultural University (approval number CAU20140910-2). All surgical operations were performed under anesthesia.

The control group were siblings born at the same time as the experimental group. The investigator was blinded to the group allocation during the experiment.

\section{Production and screening of FGF5 gene-editing sheep}

The process of production of FGF5 gene-editing sheep is shown in Fig. 1a. Templates for Cas9 mRNA and ovis FGF5 single-guide RNA transcription were constructed by our laboratory before. The target site in the ovis genome for FGF5 sgRNA is shown in Fig. 1b.

The capacity of Cas9 mRNA and FGF5 sgRNA for native gene disruption activity at its target locus was determined by DNA sequencing in microinjected individuals. The primers are as follows: forward, TGCA 


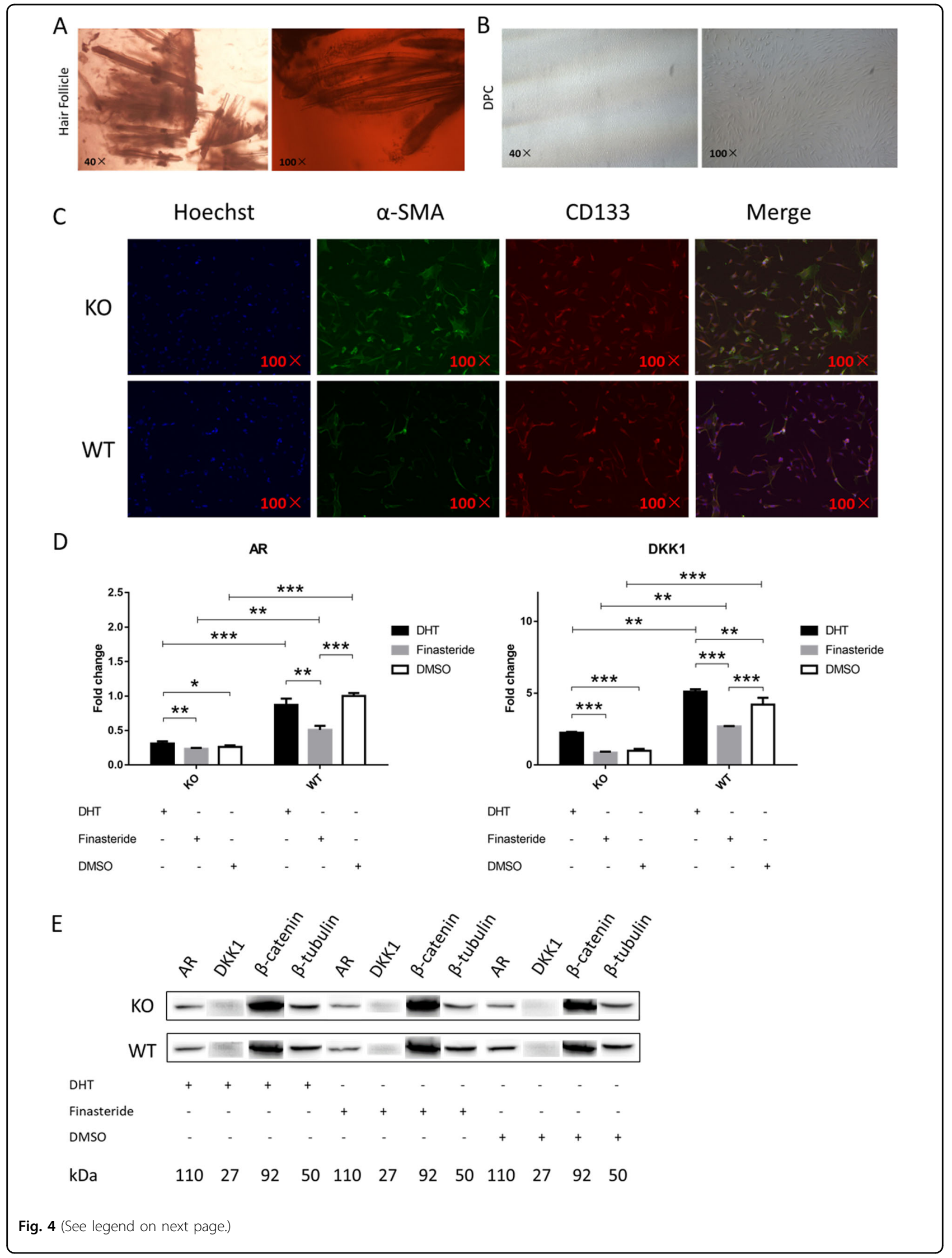


(see figure on previous page)

Fig. 4 Addition of DHT and finasteride to DPCs demonstrates the interaction between androgen and Wnt/ $\beta$-catenin. a Hair follicles of the KO and control group are obtained from skin tissue. $\mathbf{b}$ Dermal papilla cells are isolated and purified with a cloning loop. c The isolated dermal papilla cells were subjected to immunofluorescence staining. The positive a-SMA proved to be a dermal papilla cell, and CD133- positive proved that the cell was in the growth phase. $\mathbf{d}$ Changes in the relative expression levels of AR and DKK1 genes in DPCS of KO and the control group after the addition of $\mathrm{DHT}$, finasteride, and DMSO, respectively. e Changes in the relative protein levels of AR and $\beta$-catenin in DPCs of KO and the control group after the addition of DHT, finasteride, and DMSO, respectively.

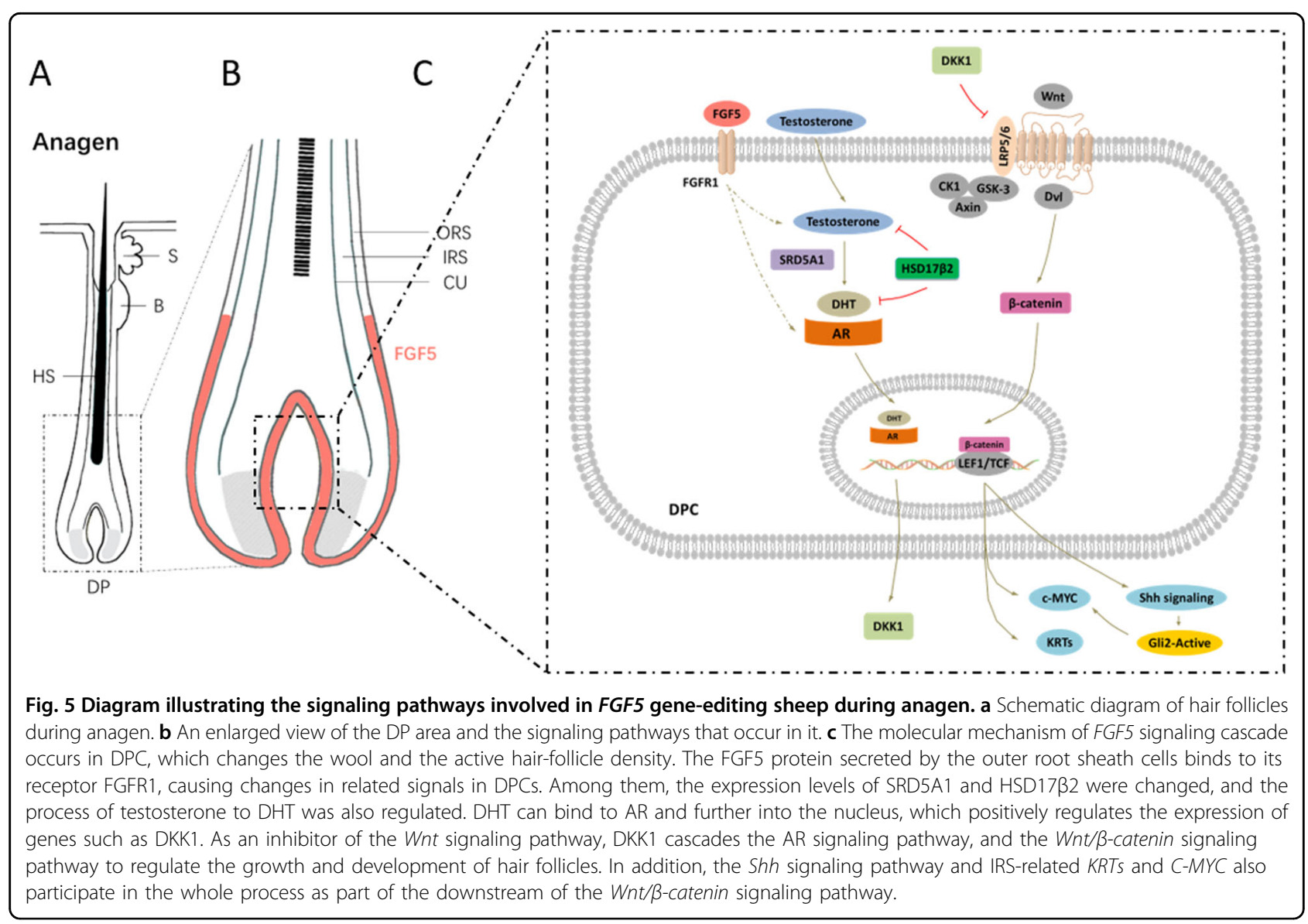

AGTTCAGGGAGCGATT and reverse, ATCCCTGTA TGCACCAAGCA. Mutations were identified by alignment of sequenced alleles to the wild-type allele. DNAMAN software was used to perform multiple alignments.

\section{Off-target detection}

To determine the site-specific cleavage of the CRISPR-Cas9 system in vivo, the potential off-target loci were searched using Cas-OFFinder (http://www. rgenome.net/cas-offinder). The selected potential offtarget sites were initially PCR-amplified and then subjected to a DNA sequencing assay. The information of the off-target loci and primer pairs used is listed in Supplementary Table S2.

\section{Changes in protein sequences and structure prediction}

The sequence of ovis FGF5 (NM_001246263.2) in NCBI (https://www.ncbi.nlm.nih.gov) was used. Changes in secondary structures were predicted by PSIPRED v3.3 (http:// bioinf.cs.ucl.ac.uk/psipred). The Phyre2 server (http://www. sbg.bio.ic.ac.uk/phyre2/html/page.cgi?id = index) was used for homology modeling and fold recognition.

\section{Measurements of associated genes by real-time PCR}

The total RNA was extracted from the skin of the KO and control groups using TRIzol reagent (Thermo Fisher Scientific, ShangHai). PrimeScrip RT reagent Kit with gDNA Eraser (Perfect Real Time) (Takara Biomedical Technology, Beijing) was used to obtain the cDNA. Real-time PCR was performed on ABI Stratagene 
Mx3000P instrument (Agilent Technologies, Santa Clara, CA, USA) using a TB Green Premix Ex Taq II (Takara Biomedical Technology, Beijing). The primer sequences used in this experiment are shown in Supplementary Table S3. The expression levels were analyzed using the $2^{\Delta \Delta \mathrm{Ct}}$ method, and normalized against $G A P D H$. Each sample was run in triplicate.

\section{ELISA and western blotting analysis}

For ELISA, skin tissue samples of the $\mathrm{KO}$ and control groups were obtained and homogenized by hand grinders, removing the supernatant after centrifugation. The ELISA procedure was performed as described in the product (TSZELISA, USA). The concentrations of androstenedione and testosterone in the skin tissue were measured using an androstenedione ELISA kit (Hermes Criterion Biotechnology, Vancouver, Canada) and a testosterone ELISA kit (Hermes Criterion Biotechnology, Vancouver, Canada) in accordance with the manufacturer's protocol.

For western blotting, equal amounts of protein of sheep skin tissue or DPCs were resolved on 12\% SDSpolyacrylamide gel (SDS-PAGE) and transferred to the polyvinylidene difluoride membrane (PVDF). After incubation with antibodies, protein bands were detected using ECL chemiluminescence (Thermo Fisher Scientific, USA). The primary antibodies were rabbit antiFGF5 antibody (Thermo Fisher Scientific, USA), goat anti-SRD5A1 antibody (Abcam, UK), rabbit antiandrogen receptor antibody (Abcam, UK), goat antiDKK1 antibody (Abcam, UK), beta-catenin polyclonal antibody (Proteintech, USA), c-MYC polyclonal antibody (Proteintech, USA), and beta tubulin monoclonal antibody (Proteintech, USA). The secondary antibodies were HRP-conjugated affinipure goat anti-rabbit IgG $(\mathrm{H}+\mathrm{L})$ (Proteintech, USA), HRP-conjugated affinipure rabbit anti-goat $\operatorname{IgG}(\mathrm{H}+\mathrm{L})$ (Proteintech, USA), and HRP-conjugated affinipure goat anti-mouse $\operatorname{IgG}(\mathrm{H}+\mathrm{L})$ (Proteintech, USA).

\section{Analytical procedures of wool phenotype of FGF5 KO sheep}

The diameters of 200 randomly chosen fiber samples were measured using an Optic Fiber Diameter Analyzer (CU-6, Beijing United Vision Technical Company, Beijing, China). Photographs were documented for $48 \mathrm{~h}$ after shaving to provide a record of wool density. Images were captured with a handheld digital microscope-HotBeauty Skin tester HtB20S. Photographs were analyzed by using the ruler to correct the actual field of view, and the densities of coarse and fine wool were calculated separately.

\section{Sacpic of hair follicles}

Sacpic is a histological method for determining the proportion of growing hair follicles in skin samples. Skin preparations of the $\mathrm{KO}$ and control groups were used to illustrate structural characteristics of follicles viewed in cross section at various stages of the hair cycle. Data from skin biopsies were also used to demonstrate quantitative estimation of follicle activity.

\section{DP cells' acquisition and culture conditions}

The skin tissues of the $\mathrm{KO}$ and control groups were digested with neutral protease (Gibco, Grand Island, NY, USA) at $37^{\circ} \mathrm{C}$ for $2 \mathrm{~h}$. The epidermis was carefully peeled off; the hair follicles were separately clustered from other tissues with a microscopic flaw. Excess tissues between hair follicles were removed by digestion with collagenase II (Gibco, Grand Island, NY, USA) at room temperature for about $30 \mathrm{~min}$. Finally, the hair follicles were transferred to the culture dish; DP cells will slowly overflow in DMEM/F-12 (Dulbecco's Modified Eagle Medium/Nutrient Mixture F12), $20 \%(\mathrm{v} / \mathrm{v})$ fetal bovine serum (FBS) supplemented with 100 units $/ \mathrm{ml}$ penicillin and $0.1 \mathrm{mg} / \mathrm{ml}$ streptomycin (Invitrogen, Carlsbad, CA, and Sigma, St. Louis, MO, USA). DPCs were further purified and extracted using a cloning loop. For all experiments, cells were used between passage three and six.

The effects of DHT and finasteride on DPCs in the KO and control groups were also determined. DPCs $\left(1.0 \times 10^{5}\right.$ cells $/ \mathrm{ml}$ in $100-\mathrm{mm}$ dishes) were seeded into $100-\mathrm{mm}$ culture dishes. After a night of adherent growth, DPCs were treated with $10^{-7} \mathrm{M}$ DHT and $10^{-5} \mathrm{M}$ finasteride (Solarbio, Beijing, China) for the next $48 \mathrm{~h}$, respectively. The media were changed every $24 \mathrm{~h}$. After 2 days of conditioned culture, DPCs were collected for the extraction of total RNA and protein.

\section{Immunofluorescence analysis}

DPCs were seeded in 96-well plates (Corning, USA) in each well. Cells were fixed with $4 \%$ paraformaldehyde for $20 \mathrm{~min}$ at room temperature, and then permeabilized with $0.3 \%$ Triton $\mathrm{X}-100$ for $10 \mathrm{~min}$ at room temperature. They were washed three times with PBS for $5 \mathrm{~min}$. After a 20-min incubation with immunol staining blocking buffer (Beyotime, Shanghai, China), cells were continuously incubated with mouse antiSMA (1:100, Abcam) and rabbit anti-CD133 (1:100, Abnova $)^{44}$ at $4{ }^{\circ} \mathrm{C}$ overnight. Then cells were washed three times with PBS followed by incubation with the secondary antibody (1:200, Proteintech) and Hoechst 33342 (1:1000) for $1 \mathrm{~h}$. Ninety-six-well plates with the fixed cells were examined using ImageXpress (Molecular Devices).

\section{Statistical analysis}

Statistical comparison of wool fineness and density was performed by the one-way ANOVA. Statistical analyses 
were performed using SAS release 9.2 (SAS Inst. Inc., Cary, NC).

\section{Acknowledgements}

We thank all the members in State Key Laboratory of Animal Nutrition, College of Animal Science and Technology, and China Agricultural University, for their help in investigating the changes in wool phenotype. This research was supported by grants from the National Transgenic Creature Breeding Grand Project (2016ZX08008-003).

\section{Author details}

'Beijing Key Laboratory of Animal Genetic Improvement, National Engineering Laboratory for Animal Breeding, Key Laboratory of Animal Genetics and Breeding of the Ministry of Agriculture, College of Animal Science and Technology, China Agricultural University, Beijing 100193, China. ${ }^{2}$ Tianjin Institute of Animal Sciences, Tianjin 300112, China. ${ }^{3}$ CAS Key Laboratory of Genome Sciences and Information, Beijing Institute of Genomics, Chinese Academy of Sciences, Beijing 100101, China

\section{Conflict of interest}

The authors declare that they have no conflict of interest.

\section{Publisher's note}

Springer Nature remains neutral with regard to jurisdictional claims in published maps and institutional affiliations.

Supplementary Information accompanies this paper at (https://doi.org/ 10.1038/s41419-020-2622-x).

Received: 3 February 2020 Revised: 15 May 2020 Accepted: 15 May 2020 Published online: 29 May 2020

\section{References}

1. Stenn, K. S. \& Paus, R. Controls of hair follicle cycling. Physiol. Rev. 81, 449 (2001).

2. Schneider, M. R., Schmidt-Ullrich, R. \& Paus, R. The hair follicle as a dynamic miniorgan. Curr. Biol. 19, R132 (2009).

3. Krause, K. \& Foitzik, K. Biology of the hair follicle: the basics. Semin. Cutan. Med. Surg. 25, 2 (2006)

4. Fuchs, E., Merrill, B. J., Jamora, C. \& DasGupta, R. At the Roots of a Never-Ending Cycle. Dev. Cell 1, 13-25 (2001).

5. Beenken, A. \& Mohammadi, M. The FGF family: biology, pathophysiology and therapy. Nat. Rev. Drug. Discov. 8, 235 (2009).

6. Hebert, J. M., Rosenquist, T., Gotz, J. \& Martin, G. R. FGF5 as a regulator of the hair growth cycle: evidence from targeted and spontaneous mutations. Cell 78, 1017 (1994)

7. Kehler, J. S. et al. Four independent mutations in the feline fibroblast growth factor 5 gene determine the long-haired phenotype in domestic cats. J. Hered. 98, 555 (2007).

8. Drögemüller, C., Rüfenacht, S., Wichert, B. \& Leeb, T. Mutations within the FGF5 gene are associated with hair length in cats. Anim. Genet. 38, 218 (2007).

9. Housley, D. J. E. \& Venta, P. J. The long and the short of it: evidence that FGF5 is a major determinant of canine 'hair'-itability. Anim. Genet. 37, 309 (2006).

10. Dierks, C., Mömke, S., Philipp, U. \& Distl, O. Allelic heterogeneity of FGF5 mutations causes the long-hair phenotype in dogs. Anim. Genet. 44, 425 (2013).

11. Legrand, R., Tiret, L. \& Abitbol, M. Two recessive mutations in FGF5 are associated with the long-hair phenotype in donkeys. Genet. Sel. Evol. 46, 65 (2014).

12. Yoshizawa, Y. et al. A 1-bp deletion in Fgf5 causes male-dominant long hair in the Syrian hamster. Mamm. Genome 26, 630 (2015).

13. $\mathrm{Yu}, \mathrm{F}$. et al. A nonsense mutation in the FGF5 gene is associated with the long-haired phenotype in domestic guinea pigs (Cavia porcellus). Anim. Genet. 49, 269 (2018).
14. Higgins, C. A. et al. FGF5 is a crucial regulator of hair length in humans. Proc. Natl Acad. Sci. USA 111, 10648 (2014).

15. Antonova, E. et al. Successful CRISPR/Cas9 mediated homologous recombination in a chicken cell line. F1000Res. 7, 238 (2018).

16. Hansmeier, N. R., Widdershooven, P. J. M., Khani, S. \& Kornfeld, J. Rapid generation of long noncoding RNA knockout mice using CRISPR/Cas9 technology. Non-Coding RNA 5, 12 (2019).

17. Hao, F. et al. Generation of cashmere goats carrying an EDAR gene mutant using CRISPR-Cas9-mediated genome editing. Int J. Biol. Sci. 14, 427 (2018).

18. Liu, Q., Yuan, Y., Zhu, F., Hong, Y. \& Ge, R. Efficient genome editing using CRISPR/Cas9 ribonucleoprotein approach in cultured Medaka fish cells. Biol. OPEN 7, 035170 (2018).

19. Petersen, B. Basics of genome editing technology and its application in livestock species. Reprod. Domest. Anim. 52, 4 (2017).

20. Xiang, G. et al. Editing porcine IGF2 regulatory element improved meat production in Chinese Bama pigs. Cell Mol. Life Sci. 75, 4619 (2018).

21. Steyer, B., Cory, E. \& Saha, K. Developing precision medicine using scarless genome editing of human pluripotent stem cells. Drug Discov. Today.: Technol. 28, 3 (2018).

22. $\mathrm{Hu}$, R. et al. RAPID COMMUNICATION: generation of FGF5 knockout sheep via the CRISPR/Cas9 system. J. Anim. Sci. 95, 2019 (2017).

23. Ceruti, J. M., Leirós, G. J. \& Balañá, M. E. Androgens and androgen receptor action in skin and hair follicles. Mol. Cell Endocrinol. 465, 122 (2018).

24. Leirós, G. J., Ceruti, J. M., Castellanos, M. L., Kusinsky, A. G. \& Balañá, M. E. Androgens modify Wnt agonists/antagonists expression balance in dermal papilla cells preventing hair follicle stem cell differentiation in androgenetic alopecia. Mol. Cell Endocrinol. 439, 26 (2017).

25. Andersson, S. \& Russell, D. W. Structural and biochemical properties of cloned and expressed human and rat steroid 5 alpha-reductases. Proc. Natl. Acad Sci. USA 87, 3640-3644 (1990).

26. Sánchez, P., Serrano-Falcón, C., Torres, J. M., Serrano, S. \& Ortega, E. 5aReductase isozymes and aromatase mRNA levels in plucked hair from young women with female pattern hair loss. Arch. Dermatol. Res. 310, 77 (2018).

27. Gao, X. et al. Functional silencing of HSD17B2 in prostate cancer promotes disease progression. Clin. Cancer Res. 25, 1291-1301 (2019).

28. Leiros, G. J., Attorresi, A. I. \& Balana, M. E. Hair follicle stem cell differentiation is inhibited through cross-talk between Wnt/beta-catenin and androgen signalling in dermal papilla cells from patients with androgenetic alopecia. Br. J. Dermatol. 166, 1035 (2012).

29. Andl, T., Reddy, S. T., Gaddapara, T. \& Millar, S. E. WNT signals are required for the initiation of hair follicle development. Dev. Cell 2, 643 (2002).

30. Lim, C. H. et al. Hedgehog stimulates hair follicle neogenesis by creating inductive dermis during murine skin wound healing. Nat. Commun. 9, 1-13 (2018).

31. Veltri, A., Lang, C. \& Lien, W. H. Concise review: Wnt signaling pathways in skin development and epidermal stem cells. Stem. Cells 36, 22 (2018).

32. Pattanayak, V. et al. High-throughput profling of off-target DNA cleavage reveals RNA-programmed Cas9 nuclease specificity. Nat. Biotechnol. 31, 839 (2013).

33. Mill, P. et al. Sonic hedgehog-dependent activation of Gli2 is essential for embryonic hair follicle development. Gene Dev. 17, 282 (2003).

34. Hsu, P. D., Lander, E. S. \& Zhang, F. Development and applications of CRISPRCas9 for genome engineering. Cell 157, 1262 (2014).

35. Karimian, A. et al. CRISPR/Cas9 technology as a potent molecular tool for gene therapy. J. Cell Physiol. 234, 12267-12277 (2019).

36. Li, W. et al. CRISPR/Cas9-mediated loss of FGF5 function increases wool staple length in sheep. FEBS J. 284, 2764 (2017).

37. Wang, $X$. et al. Disruption of FGF5 in cashmere goats using CRISPR/Cas9 results in more secondary hair follicles and longer fibers. PLOS ONE 11, e164640 (2016).

38. Millar, S. E. Molecular mechanisms regulating hair follicle development. J. Investig. Dermatol. 118, 216 (2002).

39. Kishimoto, J., Burgeson, R. E. \& Morgan, B. A. Wnt signaling maintains the hair-inducing activity of the dermal papilla. Genes Dev. 14, 1181 (2000).

40. Ito, M. et al. Wnt-dependent de novo hair follicle regeneration in adult mouse skin after wounding. Nature 447, 316 (2007). 
41. Kwack, M. H. et al. Dihydrotestosterone-inducible Dickkopf 1 from balding dermal papilla cells causes apoptosis in follicular keratinocytes. J. Investig Dermatol. 128, 262 (2008).

42. Wang, $K$. et al. Anti-proliferative activities of finasteride in benign prostate epithelial cells require stromal fibroblasts and c-Jun gene. PLOS ONE 12, e172233 (2017).
43. Armstrong, $\mathrm{K}$. et al. Upregulated FGFR1 expression is associated with the transition of hormone-naive to castrate-resistant prostate cancer. Br. J. Cancer 105, 1362 (2011).

44. Ito, Y. et al. Isolation of murine hair-inducing cells using the cell surface marker Prominin-1/CD133. J. Investig. Dermatol. 127, 1052 (2007) 